DOI: https://doi.org/10.14311/TPFM.2021.008

\title{
THERMAL DIFFUSION AND PHASE CHANGE IN A HEAT EXCHANGER
}

\author{
Gloria Faccanoni ${ }^{1}$, Cedric Galusinski ${ }^{1}$, Louis Lamerand ${ }^{1}$ \\ 1 IMATH, EA 2134, Université de Toulon, CS 60584, 83041 Toulon Cedex 9, France
}

\begin{abstract}
This work is devoted to the numerical simulation of liquid-vapour flows with phase transition in nuclear framework. We investigate the LMNC model enriched with thermal diffusion, describing the evolution of the coolant within a core of a Pressurized Water Reactor. We focus on the influence of the thermal diffusion on a simple configuration for which some analytical computations can be done and we compare two different numerical approaches.
\end{abstract}

Keywords: phase change, Stefan problem, nonlinear degenerate diffusion.

\section{Motivation}

In some applications, such as the flow in nuclear reactor cores, convection is characterized by a low Mach number, where the convective velocities are much slower than the speed of sound in the fluid. This has promoted the development of so-called low Mach number models, which filter the sound waves.

In the context of pressurized water reactor cores, an asymptotic low Mach number model, called LMNC (for Low Mach Nuclear Core), has been derived and investigated in a series of papers [1, $3,4,6]$. The model was derived through an asymptotic expansion performed in the compressible Navier-Stokes equations with an energy source term. It consists of a transport equation upon a thermodynamic variable (here the total enthalpy), of a divergence constraint upon the velocity (with a nonlinear coupling source term which underlines the dilation property of the flow) and of the momentum equation. The fluid is described by a single equation of state taking into account the phase transition by supposing that, when both vapour and liquid phases are present, they have same pressure, temperature and chemical potential.

In the present paper we are interested to study the influence of the thermal diffusion.

\subsection{Governing equation}

For some bounded domain $\Omega \subset \mathbb{R}^{3}$, the LMNC model in a 3D nonconservative formulation reads

$$
\left\{\begin{array}{l}
\partial_{t} h+\mathbf{u} \cdot \nabla h=\left(\Phi(t, \mathbf{y})+\nabla \cdot\left(\omega(h) \nabla T\left(h, p_{*}\right)\right)\right) \tau\left(h, p_{*}\right), \\
\partial_{t} \mathbf{u}+(\mathbf{u} \cdot \nabla) \mathbf{u}-\tau\left(h, p_{*}\right) \nabla \cdot \sigma(\mathbf{u})+\tau\left(h, p_{*}\right) \nabla \bar{p}=\mathbf{g}, \quad(t, \mathbf{y}) \in \mathbb{R}^{+} \times \Omega, \\
\nabla \cdot \mathbf{u}=\left.\left(\Phi(t, \mathbf{y})+\nabla \cdot\left(\omega(h) \nabla T\left(h, p_{*}\right)\right)\right) \frac{\partial \tau}{\partial h}\right|_{p},
\end{array}\right.
$$

where $\mathbf{u}$ and $h$ denote respectively the velocity field and the total enthalpy of the fluid. The specific volume $\tau$ and the temperature $T$ are related to the enthalpy through an equation of state and $\omega$ is the heat conductivity (assumed to be constant and isotropic for each phase). The power density $\Phi$ is a given function of time and space modelling the heating of the coolant fluid due to the fission reactions in the nuclear core. Finally, $\mathbf{g}$ is the gravity field and $\sigma(\mathbf{u})$ models viscous effects. We must emphasize that model is characterized by two pressure fields, which is classic in low Mach number approaches. The reference pressure $p_{*}$ is involved in the equation of state and is an average pressure (constant in time and space) within the core $\left(p_{*}=155\right.$ bar in a PWR). The dynamic pressure $\bar{p}$ appears in the momentum equation (1b) and can be considered as a perturbation around $p_{*}$. 


\subsection{Equation of state with phase transition}

The fluid can be in liquid $\kappa=\ell$ or vapour $\kappa=g$ phase or a mixture of them. We consider each phase $\kappa$ as a compressible fluid characterized by its thermodynamic properties, i.e. each phase is governed by a given $\operatorname{EoS}(h, p) \mapsto \tau_{\kappa}(h, p)$ where $h$ is the enthalpy, $p$ the pressure and $\tau$ the specific volume. In the LMNC model the mixture is supposed at saturation: when phases coexist they have the same pressure $p$, the same temperature $T$ and the same chemical potential $g$. Let us denote by $T^{\text {sat }}(p)$ the solution of the equation $g_{\ell}(T, p)=g_{g}(T, p)$ (the so called temperature at saturation). We can then define $h_{\kappa}^{\text {sat }}(p) \stackrel{\text { def }}{=} h_{\kappa}\left(T^{\text {sat }}(p), p\right)$ the enthalpy of the phase $\kappa$ at saturation.

The fluid is liquid if $h \leq h_{\ell}^{\text {sat }}\left(p_{*}\right)$, vapor if $h \geq h_{g}^{\text {sat }}\left(p_{*}\right)$ and a mixture at saturation if $h_{\ell}^{\text {sat }}\left(p_{*}\right)<$ $h<h_{\mathrm{g}}^{\text {sat }}\left(p_{*}\right)$. Thus $T\left(h, p_{*}\right)=T^{\text {sat }}\left(p_{*}\right)$ when $h_{\ell}^{\text {sat }}\left(p_{*}\right)<h<h_{g}^{\text {sat }}\left(p_{*}\right)$.

Since the thermodynamic pressure is constant in the LMNC model, from now on let us drop the dependency upon $p_{*}$. The transport-diffusion equation on the enthalpy (1a) can be expressed by

$$
\partial_{t} h+\mathbf{u} \cdot \nabla h-\tau(h) \nabla \cdot(\omega(h) \nabla T(h))=\Phi \tau(h) .
$$

Since the mixture is at saturation and the reference pressure is constant, $\nabla T=0$ when $h_{\ell}^{\text {sat }}<h<h_{\mathrm{g}}^{\text {sat }}$ and thus

$$
\nabla T(h)= \begin{cases}\frac{1}{c_{p, l}} \nabla h, & \text { if } h \leq h_{\ell}^{\text {sat }} \\ 0, & \text { if } h_{\ell}^{\text {sat }}<h<h_{g}^{\text {sat }} \\ \frac{1}{c_{p, g}} \nabla h, & \text { if } h \geq h_{g}^{\text {sat }}\end{cases}
$$

where $\left.c_{p, \kappa} \stackrel{\text { def }}{=} \frac{\partial h}{\partial T}\right|_{p}$ is the isobar heat capacity of the

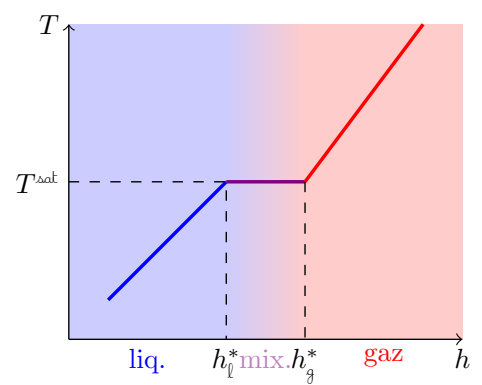
phase $\kappa=\ell$ or $g$.

\section{Toy Model for nonlinear diffusion}

By denoting

$$
\lambda(h) \stackrel{\text { def }}{=}\left\{\begin{array} { l l } 
{ \lambda _ { \ell } \stackrel { \text { def } } { = } \frac { \omega _ { \ell } } { c _ { p , \ell } } , } & { \text { if } h \leq h _ { \ell } ^ { \text { sat } } , } \\
{ 0 , } & { \text { if } h _ { \ell } ^ { \text { sat } } < h < h _ { g } ^ { \text { sat } } , } \\
{ \lambda _ { g } \stackrel { \text { def } } { = } \frac { \omega _ { g } } { c _ { p , g } } , } & { \text { if } h \geq h _ { g } ^ { \text { sat } } , }
\end{array} \quad L ( h ) \stackrel { \text { def } } { = } \left\{\begin{array}{ll}
\lambda_{\ell}\left(h-h_{\ell}^{\text {sat }}\right), & \text { if } h \leq h_{\ell}^{\text {sat }}, \\
0, & \text { if } h_{\ell}^{\text {sat }}<<h_{g}^{\text {sat }}, \\
\lambda_{g}\left(h-h_{g}^{\text {sat }}\right), & \text { if } h \geq h_{g}^{\text {sat }},
\end{array}\right.\right.
$$

so that $L^{\prime}(h)=\lambda(h)$, the enthalpy equation (2) in one dimension can be written as

$$
\partial_{t} h+v \partial_{y} h-\tau(h) \partial_{y y}^{2}(L(h))=\Phi(y, t) \tau(h), \quad \text { in } \mathbb{R}^{+} \times \mathbb{R}^{+} .
$$

In the following we consider $\tau(h)=1$ for all $h$ and the velocity $v$ and the source term $\Phi$ are chosen constant and positive. We are thus interested in the approximation of $h$ solution of the degenerate nonlinear parabolic problem:

$$
\partial_{t} h+v \partial_{y} h-\partial_{y y}^{2}(L(h))=\Phi, \quad \text { in } \mathbb{R}^{+} \times \mathbb{R}^{+}
$$

with the following initial condition and Dirichlet boundary condition:

$$
h(y, 0)=h_{\text {init }}(y) \quad h(0, t)=h_{e}<h_{\ell}^{\text {sat }} .
$$

The boundary $y=0$ corresponds to the inlet of the domain. With this assumptions the expected asymptotic enthalpy (with respect to time) is increasing with $y$.

Remark 2.1 When $\lambda_{\ell}=\lambda_{g}=0$, the steady enthalpy is $h^{\infty}(y)=h_{e}+y \Phi / v$ : the fluid is in pure liquid phase for $y \leq x_{\ell}^{*}$, in pure vapour phase for $y \geq x_{g}^{*}$ and a mixture at saturation if $x_{\ell}^{\text {sat }}<y<x_{g}^{\text {sat }}$ where

$$
x_{\ell}^{*} \stackrel{\text { def }}{=}\left(h_{\ell}^{\text {sat }}-h_{e}\right) \frac{v}{\Phi}, \quad \quad x_{g}^{*} \stackrel{\text { def }}{=}\left(h_{g}^{\text {sat }}-h_{e}\right) \frac{v}{\Phi} .
$$


The diffusion term allows the existence or not of the mixture, which can be computed explicitly for the steady solution depending on physical parameters. Nevertheless appearances or disappearances of mixture between liquid and gas phase can occur in transient regime whatever the physical parameters. This is detailed in the following subsections and leads to free boundary problems and namely the classic Stefan problem [7,8] when the transition liquid-gas occurs.

\subsection{Jump relations}

The equation (3) contains rich informations at the transition points defined by critical values of the enthalpy $h_{\ell}^{\text {sat }}$ and $h_{g}^{\text {sat }}$.

Liquid-mixture. Assuming a transition liquid/mixture at $y_{\ell}^{*}(t)$, we have

$$
\left(v-\left(y_{\ell}^{*}\right)^{\prime}(t)\right)\left(h\left(y_{\ell}^{*,+}, t\right)-h\left(y_{\ell}^{*,-}, t\right)\right)=-\lambda_{\ell} \partial_{y} h\left(y_{\ell}^{*,-}, t\right) .
$$

If $v>\left(y_{\ell}^{*}\right)^{\prime}(t)$, this implies that

$$
\left\{\begin{array}{l}
h\left(y_{\ell}^{*,-}(t), t\right)=h\left(y_{\ell}^{*,+}(t), t\right)=h_{\ell}^{\text {sat }} \\
\partial_{y} h\left(t, y_{\ell}^{*,-}(t)\right)=0
\end{array}\right.
$$

Mixture-gas. Assuming a transition mixture/gas at $y_{g}^{*}(t)$, we have

$$
\left\{\begin{array}{l}
h\left(y_{g}^{*,+}(t), t\right)=h_{g}^{\text {sat }}, \\
\left(v-\left(y_{g}^{*}\right)^{\prime}(t)\right)\left(h_{g}^{\text {sat }}-h\left(y_{g}^{*,-}, t\right)\right)=\lambda_{g} \partial_{y} h\left(y_{g}^{*,+}, t\right) .
\end{array}\right.
$$

Liquid-gas. Assuming a transition liquid/gas at $y^{*}(t) \stackrel{\text { def }}{=} y_{\ell}^{*}(t)=y_{g}^{*}(t)$, we have

$$
\left\{\begin{array}{l}
h\left(t, y^{*,-}(t)\right)=h_{\ell}^{\text {sat }} \\
h\left(t, y^{*,+}(t)\right)=h_{g}^{\text {sat }} \\
\left(v-\left(y^{*}\right)^{\prime}(t)\right)\left(h_{g}^{\text {sat }}-h_{\ell}^{\text {sat }}\right)=\lambda_{g} \partial_{y} h\left(t, y^{*,+}(t)\right)-\lambda_{\ell} \partial_{y} h\left(t, y^{*,-}(t)\right) .
\end{array}\right.
$$

This relation allows to explicit the expressions of the steady solution.

\subsection{Analytic steady solution}

From the equation written on each phase and thanks to the previous jump conditions, a simple criteria decides if the mixture exists.

- If $h_{g}^{\text {sat }}-h_{l}^{\text {sat }}>\frac{\lambda_{g}}{v} \frac{\Phi}{v}$, the mixture is present and the solution satisfies

$$
h^{\infty}(y)= \begin{cases}h_{\ell}^{\infty}(y) \stackrel{\text { def }}{=} h_{e}+\frac{\Phi}{v} y+\frac{\Phi \lambda_{\ell}}{v^{2}}\left[1-e^{\left.y v / \lambda_{\ell}\right]} e^{-y_{\ell}^{*} v / \lambda_{\ell}}\right. & \text { if } y \leq y_{\ell}^{*}, \\ h_{m}^{\infty}(y) \stackrel{\text { def }}{=} h_{\ell}^{\text {sat }}+\frac{\Phi}{v}\left(y-y_{\ell}^{*}\right) & \text { if } y_{\ell}^{*} \leq y<y_{g}^{*}, \\ h_{g}^{\infty}(y) \stackrel{\text { def }}{=} h_{\ell}^{\text {sat }}+\frac{\Phi}{v}\left(y-y_{g}^{*}\right) & \text { if } y \geq y_{g}^{*} .\end{cases}
$$

The position $y_{\ell}^{*}$ is implicitly defined by $h_{\ell}^{\infty}\left(y_{\ell}^{*}\right)=h_{\ell}^{\text {sat }}$. At $y_{g}^{*}$, the jump is in the mixture region and

$$
h_{g}^{\text {sat }}-h_{m}^{\infty}\left(y_{g}^{*}\right)=\frac{\lambda_{g}}{v} \frac{\Phi}{v}<h_{g}^{\text {sat }}-h_{\ell}^{\text {sat }}
$$

then follows $y_{g}^{*}$ by the relation

$$
y_{g}^{*}=y_{\ell}^{*}+\frac{v}{\Phi}\left(h_{g}^{\text {sat }}-h_{\ell}^{\text {sat }}\right)-\frac{\lambda_{g}}{v} .
$$


It follows that gas diffusion reduces the mixture region for steady solution since

$$
\left(y_{g}^{*}-y_{\ell}^{*}\right)=\left(x_{g}^{*}-x_{\ell}^{*}\right)-\frac{\lambda_{g}}{v} .
$$

- If $h_{g}^{\text {sat }}-h_{l}^{\text {sat }} \leq \frac{\lambda_{g}}{v} \frac{\Phi}{v}$, the mixture does not exist, the transition point is $y^{*}=y_{l}^{*}=y_{g}^{*}$ and the two phases solution satisfies

$$
h^{\infty}(y)= \begin{cases}h_{\ell}^{\infty}(y) \stackrel{\text { def }}{=} h_{e}+\frac{\Phi}{v} y+\left[\left(h_{g}^{\text {sat }}-h_{\ell}^{\text {sat }}\right)-\left(\frac{\lambda_{g}}{v}-\frac{\lambda_{l}}{v}\right) \frac{\Phi}{v}\right]\left[1-e^{y v / \lambda_{l}}\right] e^{-y^{*} v / \lambda_{l}} & \text { if } y<y^{*} \\ h_{g}^{\infty}(y)=h_{g}^{*}+\frac{\Phi}{v}\left(y-y^{*}\right) & \text { if } y>y^{*}\end{cases}
$$

with $y^{*}$ implicitly defined by $h_{\ell}^{\infty}\left(y^{*}\right)=h_{\ell}^{\text {sat }}$.

This can be summarized on the figure 1 where the mixture region is always smaller than the one obtained without diffusion, i.e. $y_{g}^{*}-y_{\ell}^{*} \leq x_{g}^{*}-x_{\ell}^{*}$. On the figure 1a, data are chosen so that $h_{g}^{\text {sat }}-h_{\ell}^{\text {sat }}>\lambda_{g} \Phi / v^{2}$ in order to produce a mixture (diffusion can be neglected if $\lambda_{g} \ll$ $\left.\left(h_{g}^{\text {sat }}-h_{\ell}^{\text {sat }}\right) v^{2} / \Phi\right)$. On the contrary, the inverse inequality $h_{g}^{\text {sat }}-h_{\ell}^{\text {sat }} \leq \lambda_{g} \Phi / v^{2}$ leads to the steady solution on the figure $1 b$ where the mixture region does not exist.

\section{Numerical experiments}

The interest to write models for each phase linked by jump relations (5)-(7) or (8) can help to solve linear PDEs on each phase and then to move boundaries with respect to the jump relations. This is the classic way to solve Stefan-like problem. If it is easily implemented for 1D problem, it is more complicated for 2D and 3D problem. The main obstacle occurs, even for the $1 \mathrm{D}$ case, when the appearance or disappearance of phase has to be managed. This leads to choose numerical scheme to approach the nonlinear unified PDE (3). The goal of this section is to introduce unsteady numerical reference solutions based on the Stefan-like approach and to compare it with the numerical solutions of the unified model. If the right jump relations are well implicitly contained in such a numerical scheme, the phase transition should move with an accurate velocity.

\subsection{Numerical scheme for Stefan-like formulation}

- If $h_{g}^{\text {sat }}-h_{l}^{\text {sat }}>\frac{\lambda_{g}}{v} \frac{\Phi}{v}$, we assume that the initial enthalpy $h_{\text {init }}$ is increasing and defines three phase regions (liquid on the domain $\left(0, y_{\ell}^{*}(0)\right)$, mixture in $\left(y_{\ell}^{*}(0), y_{g}^{*}(0)\right)$ and vapour in $\left.\left(y_{g}^{*}(0), L\right)\right)$. The jump relations and equations are solved and splitted in the following order to provide an accurate unsteady solution.

- For a fixed positive time $t$, on the domain $\left(0, y_{\ell}^{*}(t)\right)$ the liquid phase solves a linear transport diffusion with overdetermined boundary conditions (6). An iterative process on the position $y_{0}^{*}(t)$ allows to solve such a linear problem with a simple finite difference scheme. Three iterations are sufficient to catch the overdetermined boundary condition. The velocity of the interface can then be computed in a discrete way thanks to the previous position $y_{\ell}^{*}$ at the previous time step.

- The gas transition position $y_{g}^{*}(t)$ is predicted thanks to the jump relation (7) defining the velocity at the previous time step and position is obtained by a discrete time integration. Then, the linear transport diffusion equation is solved on the domain $\left(y_{g}^{*}(t), L\right)$ with the same scheme as for the liquid phase with Dirichlet boundary condition at the left boundary and inhomogeneous Neuman boundary condition at the right boundary.

- Then, on the mixture region $\left(y_{\ell}^{*}(t), y_{g}^{*}(t)\right)$, the transport diffusion is solved thanks to the inlet boundary $h\left(y_{\ell}^{*}(t), t\right)=h_{\ell}^{\text {sat }}$. The value of the enthalpy at the outlet $h\left(y_{g}^{*}(t), t\right)$ will help to compute the jump on the enthalpy to provide the new velocity of $y_{g}^{*}$. 
- If $h_{g}^{\text {sat }}-h_{\ell}^{\text {sat }} \leq \frac{\lambda_{g}}{v} \frac{\Phi}{v}$, we assume that the initial enthalpy $h_{\text {init }}$ is increasing and defines two phases (liquid on the domain $\left(0, y^{*}(0)\right)$ and gas in $\left(y^{*}(0), L\right)$ ), jumping at $y^{*}$ from $h_{\ell}^{\text {sat }}$ to $h_{g}^{\text {sat. }}$. Then the free boundary problem is solved by moving the interface $y^{*}(t)$ with respect to the jump relation (8) based on slope of enthalpy on each side of the interface.

- On the liquid domain $\left(0, y^{*}(t)\right)$, the transport diffusion is solved with Dirichlet boundary condition on both sides (namely $h_{\ell}^{\text {sat }}$ at $\left.y^{*}(t)\right)$ ) .

- On the gas domain $\left(y^{*}(t), L\right)$, the transport diffusion is solved with Dirichlet boundary condition at $\left.y^{*}(t)\right)$ and a Neuman boundary condition on the artificial outlet at $L$, instead of the infinite domain.

Figure 2 displays the numerical solution from the initial condition (dotted) to the steady solution (dashed). Figure 2a displays the case where the solution contains all the time liquid, mixture and vapour phases; Figure $2 \mathrm{~b}$ the case where the solution presents always liquid and vapour phases without mixture. Not appearance or disappearance of a pure phase or the mixture is taken into account.

We display on Figure 3 the numerical speed of the interfaces on three increasingly refined grids (200-400-800) establishing the grid convergence. No spurious oscillations appear on these speeds and provide accurate positions of phase transition. Figures $3 \mathrm{a}$ and $3 \mathrm{~b}$ display the interfaces liquid/mixture and mixture/vapour respectively for the test in figure 2a; figure 3c the interface liquid/vapour for the test in figure $2 \mathrm{~b}$.

In the following we consider this unsteady numerical solution based on the Stefan-like approach as a reference solution for the numerical solutions of the unified model.

\subsection{Numerical scheme for unified model}

The model (3) written for all phases whatever the initial enthalpy is much more general than the previous free boundary formulation and can be solved with nonlinear fully implicit time discretization,

$$
\frac{h^{n+1}-h^{n}}{\delta t}+v \partial_{y} h^{n+1}-\partial_{y y}^{2}\left(L\left(h^{n+1}\right)\right)=\Phi
$$

This approach is chosen for example in [5] associated to some restrictions on the spatial discretization referred to gradient scheme. These restrictions do not concern some simple finite difference schemes written on regular grids. It is what is chosen here for $1 \mathrm{D}$ but also $2 \mathrm{D}$ codes. Nevertheless, if the scheme convergence can be proved for weak norms ( $L^{2}$ norm for time and space), we are concerned with the ability of such a scheme to catch the discrete jump relations and then to make evolve transition points (implicitly defined by the enthalpy value) at the accurate velocity.

Figure 4 displays the numerical solution from the initial condition $h_{\text {init }}(y) \equiv h_{e}<h_{\ell}^{\text {aat }}$ (green dotted) to the steady solutions. In the beginning the domain is filled with liquid phase. After some instant the mixture phase appears. When the gas phase appears the jump respect the condition $h\left(y_{g}^{*,+}(t), t\right)=h_{g}^{\text {sat }}$ and increases up to the asymptotic jump (9). The appearance of phase along time could not be simulated with the free boundary problem approach and constitutes the main advantage of the unified model. On the left figure we display the case when the steady solution contains liquid, mixture and gas, on the right figure the steady solution contains only liquid and gas phase without mixture. Notice that in the last case the mixture is present along the transition and then disappears before that the solution converges to the asymptotic one.

Figure 5 displays numerical convergence for the fully implicit scheme. The unsteady three phases solution of reference is constructed thanks to the numerical solution of the free boundary problem on a fine grid. The plot on the left shows the $L^{2}(0, L)$ norm error along the time for different grid size. The plot on the right displays the $L^{2}((0, L) \times(0, T))$ norm error with respect to the grid size and shows a convergence at order 0.7 .

The grid convergence for liquid/mixture interface position $y_{\ell}^{*}(t)$ is established at order 0.65 ; the grid convergence for mixture/gas interface position $y_{g}^{*}(t)$ is at order 0.7 .

The enthalpy jump in $y_{g}^{*}(t)$ is computed with an error around $10-20 \%$ (even on fine grids) showing the limit of the accuracy of such an approach. In the same way, no numerical convergence can be established for the speed of transition points. 


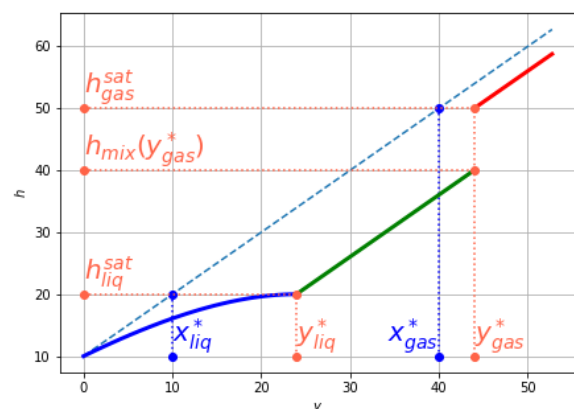

(a) Steady solution with liquid, mixture and gas.

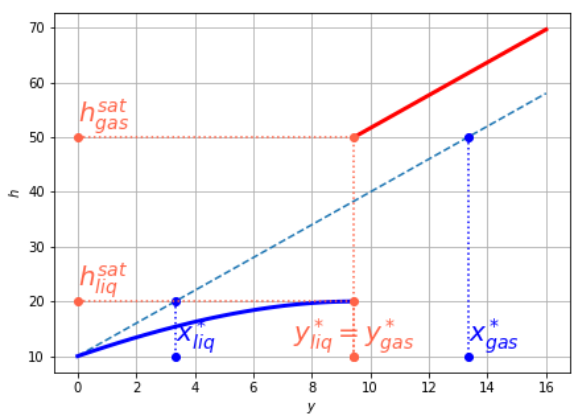

(b) Steady solution with liquid and gas (without mixture).

Figure 1: Steady solution with diffusion (with a jump at $y=y_{g}^{*}$ ) compared to the steady solution without diffusion (dashed).

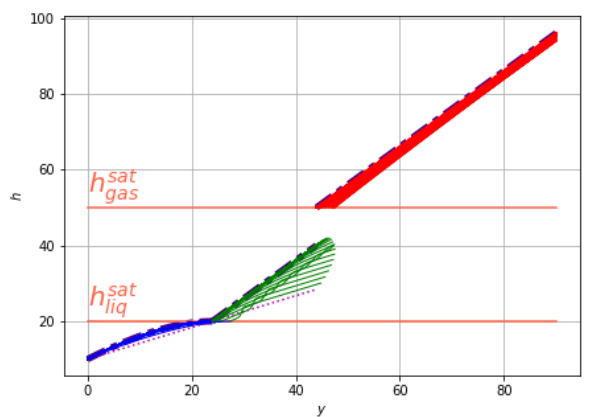

(a) Solution with liquid, mixture and gas.

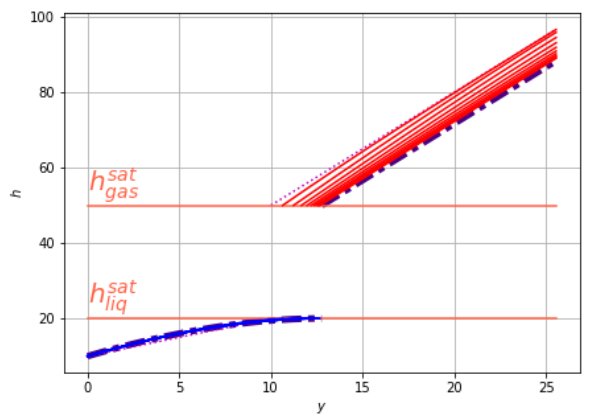

(b) Solution with liquid and gas (without mixture).

Figure 2: Dynamical solution (initial enthalpy is dotted) compared to the steady solution (dashed) based on Stefan-like formulation.

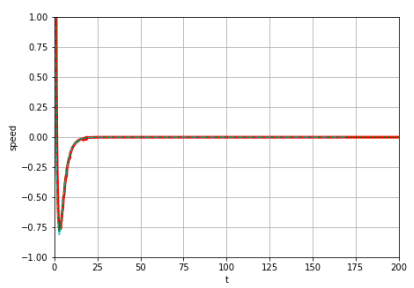

(a) Liquid/mixture interface: $t \mapsto\left(y_{\ell}^{*}\right)^{\prime}(t)$

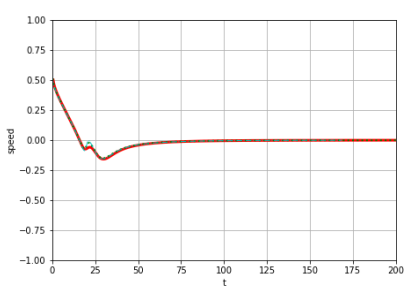

(b) Mixture/gas interface: $t \mapsto\left(y_{g}^{*}\right)^{\prime}(t)$

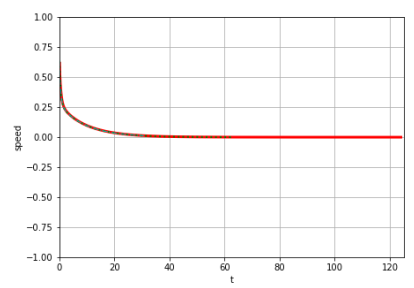

(c) Liquid/gas interface: $t \mapsto\left(y^{*}\right)^{\prime}(t)$

Figure 3: Speed of the interfaces 
This lower accuracy of the nonlinear implicit scheme compare to the free boundary approach should not obscure the generality and the simplicity of such a scheme able to handle appearance and disappearance of phases. Furthermore, the computational cost is slightly lower with the nonlinear implicit scheme.

Figure 6 displays a $2 \mathrm{~d}$ simulation of the enthalpy solution with a radial velocity field. The radial solution, computed on a 2D cartesian grid, shows similar behavior as for the 1D case with phase change occuring along time, increasing solution in the radial direction and a jump of the enthalpy downstream of the gas. The colors on the surface plot are blue for liquid, green for mixture and red for gas.

\section{Conclusions}

This study shows the influence of the thermal diffusion on the phase change in a channel with a forced flow at the inlet and a uniform heat input. The inlet is forced with a liquid phase and the phase change occurs downstream due to the energy deposit. Without diffusion, the mixture zone is always present but can be highly impacted by diffusion. The diffusion reduces this area and makes it disappear if it is sufficiently strong. The criterion for mixture disappearance relates to the gas diffusion coefficient and is even explicit for the steady flow, depending on the deposit energy $\Phi$, the velocity of the flow $v$ and the critical values of the enthalpy for the phase changes:

$$
\lambda_{g}>\frac{v^{2}}{\Phi}\left(h_{g}^{\text {sat }}-h_{\ell}^{\text {sat }}\right)
$$

When the gas diffusion coefficient is negligible compare to the above critical value, mixture area is close to the one without diffusion and diffusion can reasonably be neglected. If not, the degenerate nonlinear diffusion term should be taken into account with a fully implicit scheme. This choice is based on the simplicity of implementation and the satisfying accuracy of such an approach for transition points.

In the continuity of this work, in a future development, the implementation of the degenerate nonlinear diffusion term should be extended with a variable density depending on the enthalpy. This step does not add numerical difficulty. Additional numerical difficulties occurs for the construction of scheme on general meshes. The extension of this work could be done following [2] in order to avoid spurious oscillations in the mixture area.

The main extension concerns then the full LMNC model with additional thermal diffusion.

Acknowledgments. This work has been achieved within the framework of the M2SIR project, financially supported by CNRS AAP "NEEDS 2020", and its partners Andra, BRGM, CEA, EDF, Framatome, IRSN et Orano. 


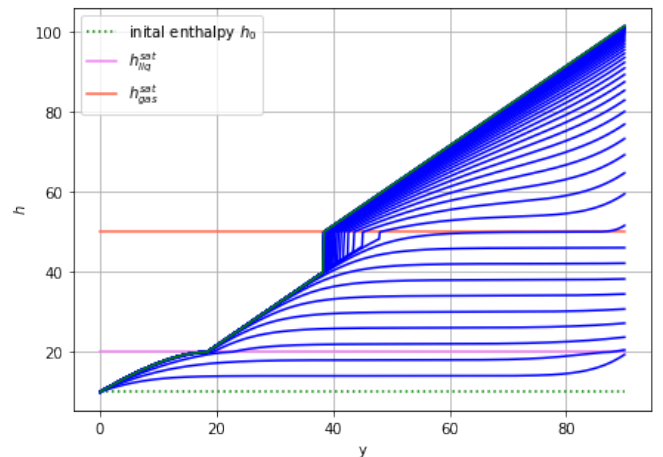

(a) Solution with asymptotic liquid, mixture and gas.

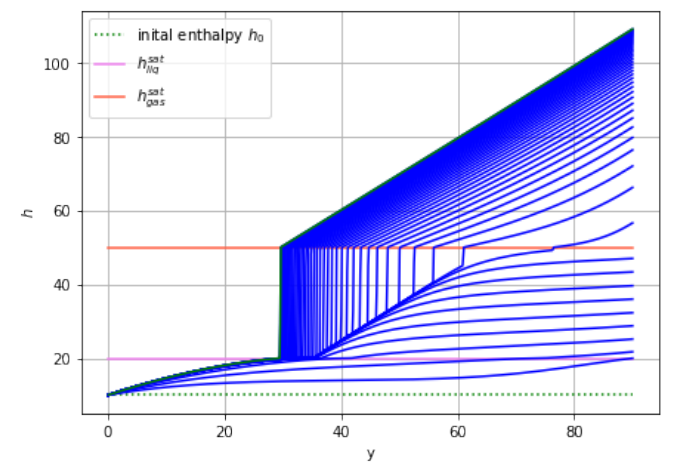

(b) Solution with asymptotic liquid and gas (without asymptotic mixture).

Figure 4: Enthalpy with respect to time

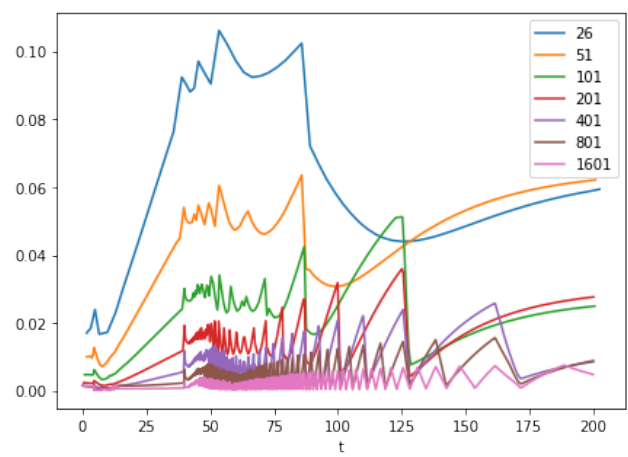

(a) $L_{y}^{2}$ Enthalpy error with respect to time

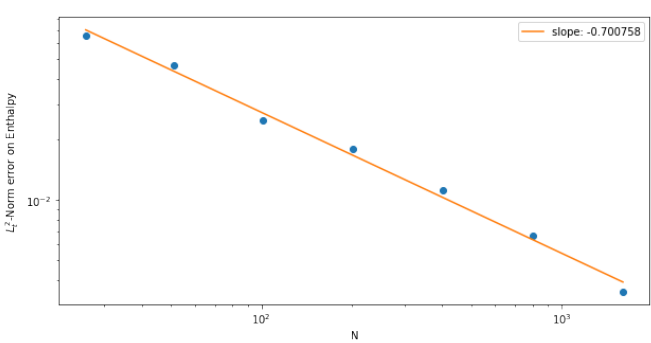

(b) Grid convergence on enthalpy (averaged $L_{t, y}^{2}$ )

Figure 5: Numerical Convergence

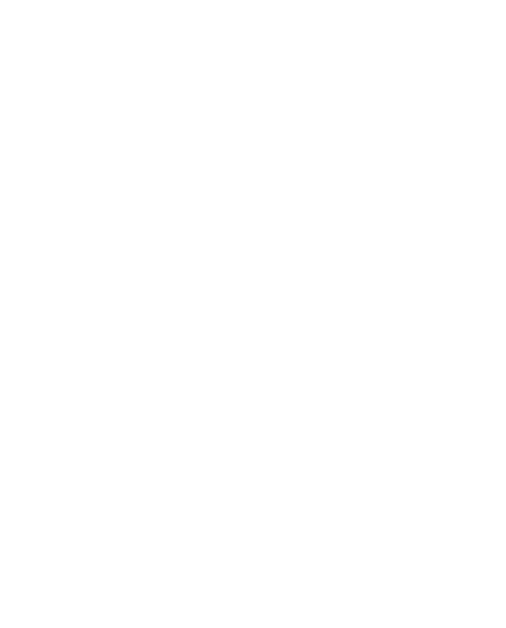

Figure 6: Simulation for the 2D unified model 


\section{References}

[1] Bernard, M., Dellacherie, S., Faccanoni, G., Grec, B. \& Penel, Y.: Study of a low Mach nuclear core model for two-phase flows with phase transition i: stiffened gas law. ESAIM: Mathematical Modelling and Numerical Analysis. vol. 48 (2014). pp. 1639-1679. doi: 10.1051/m2an/2014015. URL https://hal.archives-ouvertes.fr/hal-00747616.

[2] Cancès, C. \& Guichard, C.: Convergence of a nonlinear entropy diminishing control volume finite element scheme for solving anisotropic degenerate parabolic equations. Mathematics of Computation. vol. 85 no. 298 (2016). pp. 549-580.

[3] Dellacherie, S.: On a low Mach nuclear core model. In ESAIM:Proc: volume 35: pp. 79-106 (2012).

[4] Dellacherie, S., Faccanoni, G., Grec, B. \& Penel, Y.: Accurate steam-water equation of state for two-phase flow LMNC model with phase transition. Applied Mathematical Modelling. vol. 65 (2019). pp. 207-233. doi: 10.1016/j.apm.2018.07.028. URL https://hal . archives-ouvertes.fr/hal-01111730.

[5] Eymard, R., Féron, P., Gallouët, T., Herbin, R. \& Guichard, C.: Gradient schemes for the stefan problem. International Journal On Finite Volumes. (2013). pp. Volume-10.

[6] Faccanoni, G., Galusinski, C. \& Rafiou, M.: Numerical simulations of a low Mach number model in heat exchanger. In Šimurda, D. \& Bodnár, T., editors, Topical Problems of Fluid Mechanics 2018: (2018). URL https://hal-univ-tln.archives-ouvertes.fr/hal-01688125.

[7] Kutluay, S., Bahadir, A. \& Özdeş, A.: The numerical solution of one-phase classical Stefan problem. Journal of computational and applied mathematics. vol. 81 no. 1 (1997). pp. 135144.

[8] Rubinšteĭn, L.: The Stefan problem: volume 8. American Mathematical Soc. (2000). 